\title{
Controlling Quantum Confinement and Magnetic Doping of Cesium Lead Halide Perovskite Nanocrystals
}

\author{
Yitong Dong, David Parobek, and Dong Hee Son ${ }^{\dagger}$ \\ Department of Chemistry, Texas A\&M University, College Station, Texas 77843, USA \\ (Received August 27, 2018; Accepted September 15, 2018)
}

\begin{abstract}
Cesium lead halide $\left(\mathrm{CsPX}_{3}\right)$ nanocrystals have emerged as a new family of semiconductor nanomaterials that can outperform existing semiconductor nanocrystals owing to their superb optical and charge transport properties. Although these materials are expected to have many superior properties, control of the quantum confinement and isoelectronic magnetic doping, which can greatly enhance their optical, electronic, and magnetic properties, has faced significant challenges. These obstacles have hindered full utilization of the benefits that can be obtained by using $\mathrm{CsPb}_{3}$ nanocrystals exhibiting strong quantum confinement or coupling between exciton and magnetic dopants, which have been extensively explored in many other semiconductor quantum dots. Here, we review progress made during the past several years in tackling the issues of introducing controllable quantum confinement and doping of $\mathrm{Mn}^{2+}$ ions as the prototypical magnetic dopant in colloidal $\mathrm{CsPbX}_{3}$ nanocrystals.
\end{abstract}

Key words : Lead halide perovskite, Quantum dot, Magnetic doping

\section{Introduction}

$S^{2}$ emiconducting perovskite nanocrystals (NCs) have attracted much attention in recent years owing to their superb light-emitting properties and facile chemical tunability of the bandgap, making them particularly suitable for applications where they act as a photon ${ }^{1,2)}$ or charge carrier source. ${ }^{3-5)}$ Among various perovskite NCs, those in the cesium lead halide $\left(\mathrm{CsPbX}_{3}\right)$ system are among the most extensively explored prototypical perovskites owing to their relative ease of synthesis and potential for outperforming more commonly used II-VI or IV-VI semiconductor NCs in various applications. In particular, their ability to harvest photons to create charge carriers, transport them long distances, ${ }^{6}$ ) and emit photons ${ }^{7,8)}$ are superior to those of many known semiconductor NCs. Fig. 1 shows the crystal structure of $\mathrm{CsPbX}_{3}$, transmission electron microscopy (TEM) images of colloidal NCs of $\mathrm{CsPb}_{3}$, and a photograph showing the color-tunable fluorescence obtained by varying the halide composition..$^{9)}$ Considering the successful application of various colloidal semiconductor NCs in recent decades in solar cells, ${ }^{10-14)}$ optoelectronics, ${ }^{15-17)}$ photonics, and biological applications, ${ }^{18-20)}$ one can easily see the potential of this new family of semiconductor NCs.

Here we will focus on colloidal NCs of $\mathrm{CsPbX}_{3}$, with particular emphasis on controlling the quantum confinement and introducing a magnetic dopant into the host NC lattice. Without question, quantum confinement of the exciton is

${ }^{\dagger}$ Corresponding author: Dong Hee Son

E-mail : dhson@chem.tamu.edu

Tel : +1-979-458-2990 (a)
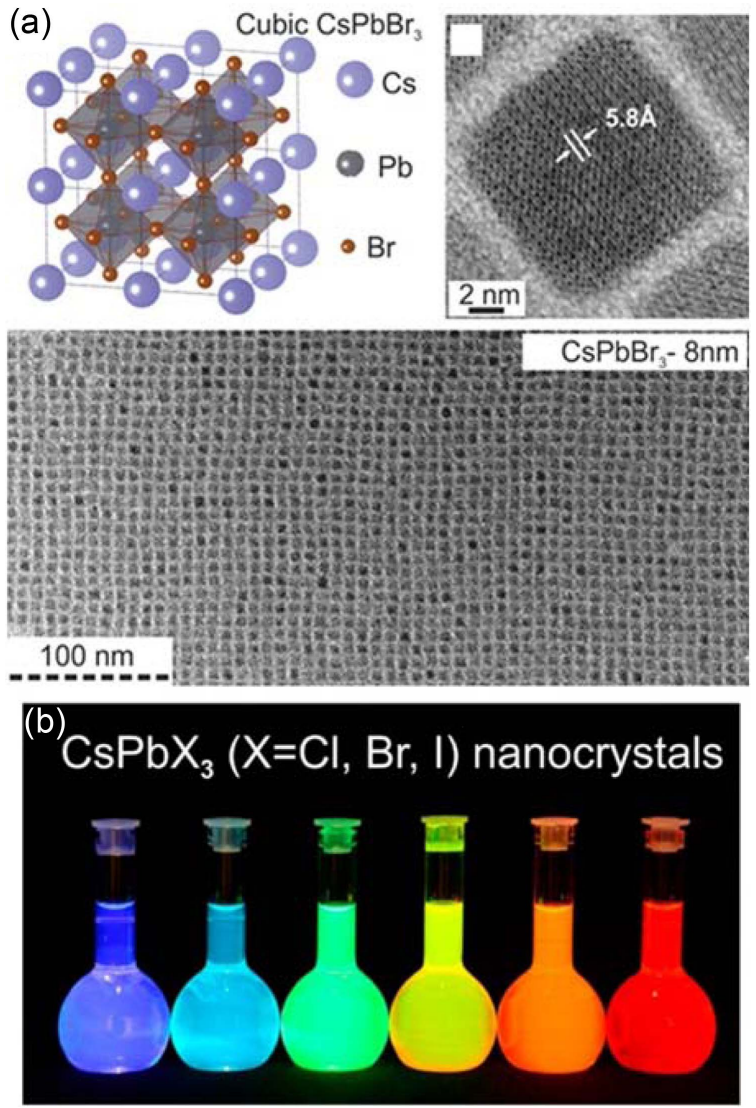

Fig. 1. (a) Crystal lattice structure and TEM images of CsP$\mathrm{bX}_{3}$ perovskite nanocrystals for $\mathrm{X}=\mathrm{Br}$. (b) Photograph of colloidal solutions of $\mathrm{CsPbX}_{3}$ nanocrystals under UV light, which exhibit tunable luminescence achieved by varying the halide composition. Images were modified with permission from Ref. 9. 
the most extensively explored phenomenon in various semiconductor NCs to obtain new properties unique to nanoscale semiconductor materials as distinct from their bulk counterparts. ${ }^{21)}$ Furthermore, isoelectronic doping of paramagnetic ions into semiconductor NCs provides access to new optical, $^{22,23)}$ electronic, ${ }^{24,25)}$ and magnetic properties ${ }^{26)}$ not present in typical semiconductor NCs through exchange coupling between the exciton and dopant. However, introducing controlled quantum confinement and doping paramagnetic transition metal ions into $\mathrm{CsPb}_{3} \mathrm{NCs}_{\text {have been }}$ significantly more challenging than in many other semiconductor materials. Only very recently has meaningful progress been made to overcome the obstacles in performing these important tasks, enabling full exploration of the potential of this new type of semiconductor NC. ${ }^{27-29)}$ Here, we review recent progress in the synthesis of colloidal quantum dots (QDs) of $\mathrm{CsPbX}_{3}$ and $\mathrm{Mn}$ doping of various $\mathrm{CsPbX}_{3}$ nanostructures.

\section{Strongly Quantum-Confined $\mathrm{CsPbX}_{3} \mathrm{NCs}$}

In the typical hot injection synthesis condition, $\mathrm{CsPbX}_{3}$ NCs with an edge length of $>10 \mathrm{~nm}$ are produced with a relatively broad size distribution. ${ }^{9)}$ For $\mathrm{CsPbBr}_{3}$, the exciton Bohr radius is $3.5 \mathrm{~nm}$, so quantum confinement of the exciton is weak. For this reason, tuning of the bandgap in CsP$\mathrm{bX}_{3}$ NCs was achieved primarily by varying the halide $(\mathrm{X})$ composition and changing the bulk band edge level rather than varying the quantum confinement of the exciton, in contrast to the procedure for QDs with a size-tuned bandgap. The post-synthesis anion exchange reaction was often used for this purpose..$^{30,31)}$

Although it is still useful to vary the absorption and emission energy in $\mathrm{CsPbX}_{3}$ via anion exchange, other material properties that result from confinement of the exciton require a controllable NC size. For instance, the exciton binding energy, ${ }^{32-34)}$ the linewidth of fluorescence emission dictated by electron-phonon coupling, ${ }^{35)}$ and the Rashba effect $^{36)}$ prominently observed in various lead halide perovskite NCs are expected to exhibit significant dependence on the quantum confinement. To explore such properties without encountering ensemble heterogeneity and utilize them in applications, the development of a robust method for the synthesis of size-controlled NCs in the quantum-confined regime is necessary. Although post-synthesis separation, often involving tedious multistage centrifugation of the heterogeneous NC sample, can improve the ensemble homogeneity, ${ }^{37,38)}$ it is a rather inefficient approach to preparing uniform perovskite QDs for practical applications.

Much effort has been made recently to extend the size range of $\mathrm{CsPbX}_{3} \mathrm{NCs}$ into the strongly confined regime. However, the usual kinetic control (i.e., control of the growth via the reaction time) widely utilized for the synthesis of various other semiconductor NCs has not been very (a)
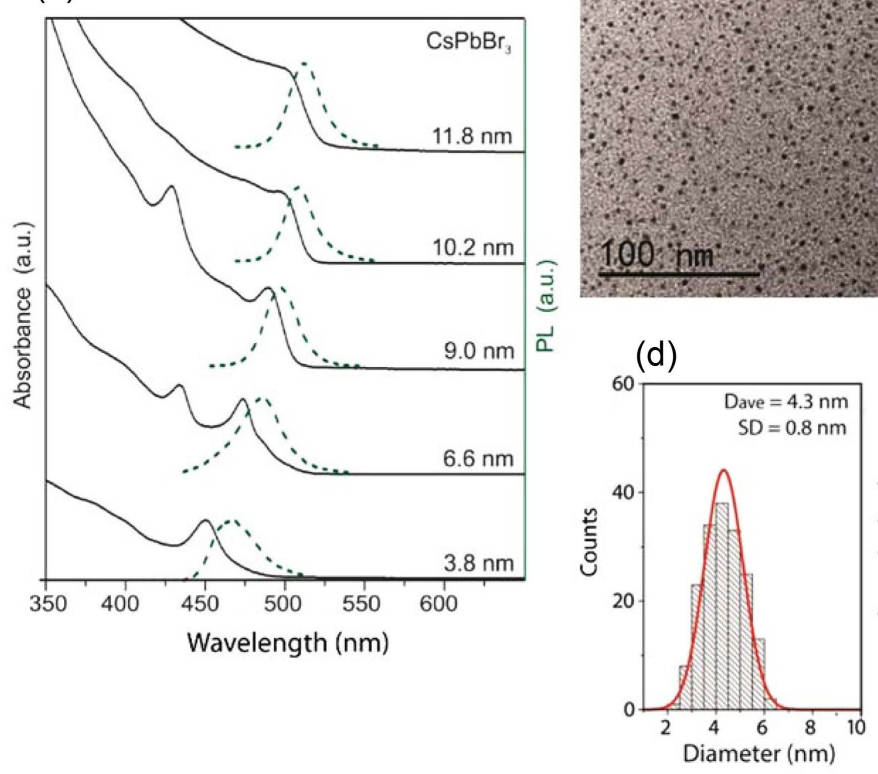


(e)

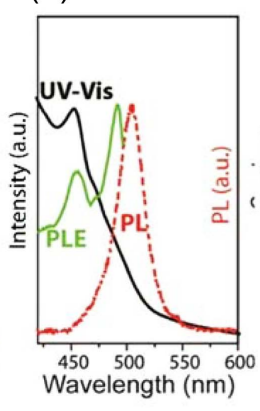

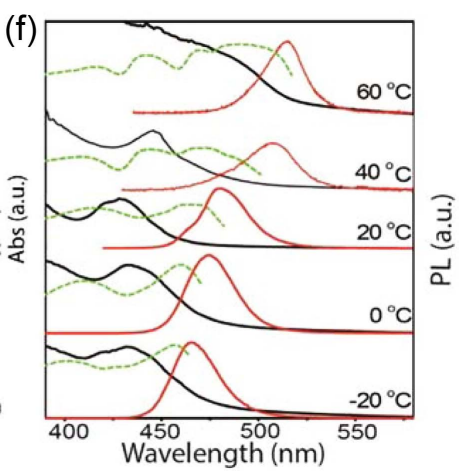

Fig. 2. (a) Absorption and photoluminescence (PL) spectra of QDs acquired by decreasing the reaction temperature. Images were modified with permission from Ref. 9. (b) and (c) TEM images of QDs made by ligand-mediated room temperature synthesis. (d) Size distribution of QDs shown in (b) and (c). (e) Absorption, PL, and PLE spectra of QDs acquired by ligandmediated method showing multiple features resulting from ensemble inhomogeneity. (f) Absorption and PL spectra of QDs acquired by the ligand-mediated method at various temperatures. Images were modified with permission from Ref. 43. 
effective for size control of $\mathrm{CsPbX}_{3} \mathrm{NCs}$ owing to the rapid reaction conditions under typical hot injection synthesis. ${ }^{9)}$ Furthermore, fast defocusing of the size after hot injection often resulted in a poor size distribution in ensembles of the NCs. ${ }^{39)}$ One strategy that has been used to gain more control over the size of the NCs in the confined regime is to lower the reaction temperature. Generally, the NC size decreased with decreasing reaction temperature, giving access to more strongly confined QDs. ${ }^{40-42)}$ However, the relatively large size distribution and contamination by NCs with other morphologies, such as nanoplatelets, remained an issue as reflected in the optical spectra exhibiting multiple excitonic peaks from different species (Fig. 2a). Another approach that has been taken to control the size is the use of different ligands (Fig. 2b-f). ${ }^{43)}$ The exciton absorption peak from the nanoplatelets was often prominently superimposed on the absorption from the nanocubes, whereas the nanoplatelets leave a less distinct spectroscopic signature in the luminescence spectrum owing to their relatively low emission quantum yield compared to the bright nanocubes. Recently, Manna and coworkers reported that the size of $\mathrm{CsPbBr}_{3}$ perovskite nanocubes can be reduced to ca. $4 \mathrm{~nm}$ by varying the composition or the amount of the ligands they used, specifically, the ratio of oleic acid to oleylamine (Fig. 3a). ${ }^{44)}$ The size was reduced by suppressing the rapid ripening rate of the perovskite NCs during growth by reduc- ing the quantity of ligands, which the authors considered to be responsible for ripening of the NCs. Because of the difference in the rate of ripening of perovskite NCs with different halides, size control via the ligand could not be extended to $\mathrm{CsPbX}_{3}$ perovskite NCs with $\mathrm{X}=\mathrm{I}$. Recently, Pradhan and coworkers reported that the size of $\mathrm{CsPbBr}_{3} \mathrm{NCs}$ can be reduced by adding an oleylamine- $\mathrm{HBr}$ mixture to the reactant. The size of the NCs could be continuously tuned by varying the amount of the oleylamine- $\mathrm{HBr}$ mixture (Fig. $3 b){ }^{45)}$ They proposed that the size control mechanism is competition between $\mathrm{Cs}^{+}$and protonated ligands during growth of the NCs. All these methods rely on additional means to control the growth kinetics; therefore, the intrinsic heterogeneity of the size limits the spectroscopic homogeneity, which is crucial for various optical applications of this material.

Very recently, Son and coworkers developed a new synthesis approach that can produce strongly quantum-confined $\mathrm{CsPbX}_{3}$ QDs with an unprecedented level of control over the size with ensemble uniformity. ${ }^{46)}$ In contrast to the majority of other synthesis methods, which attempt to control the kinetic behavior of the reaction, the new approach developed by Son's group used the thermodynamic equilibrium of halide ions to control the size. Fig. 3 compares the optical spectra and TEM images of $\mathrm{CsPbBr}_{3}$ QDs synthesized by other methods exploiting control of the kinetics (a)

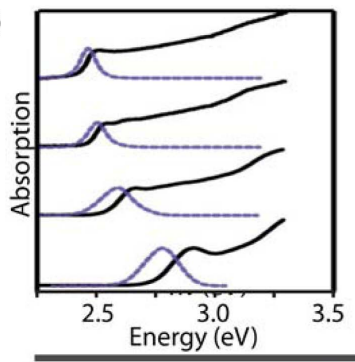

(b)

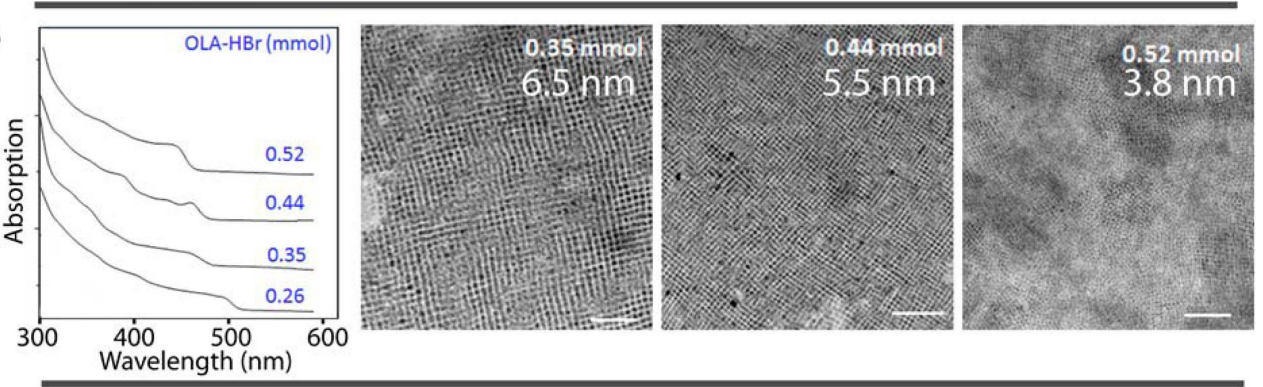

(c)

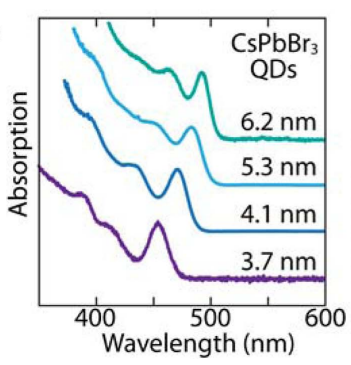

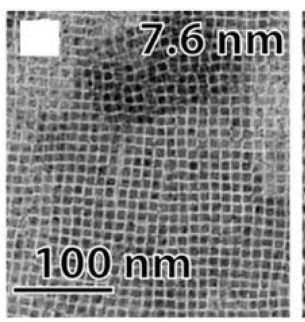
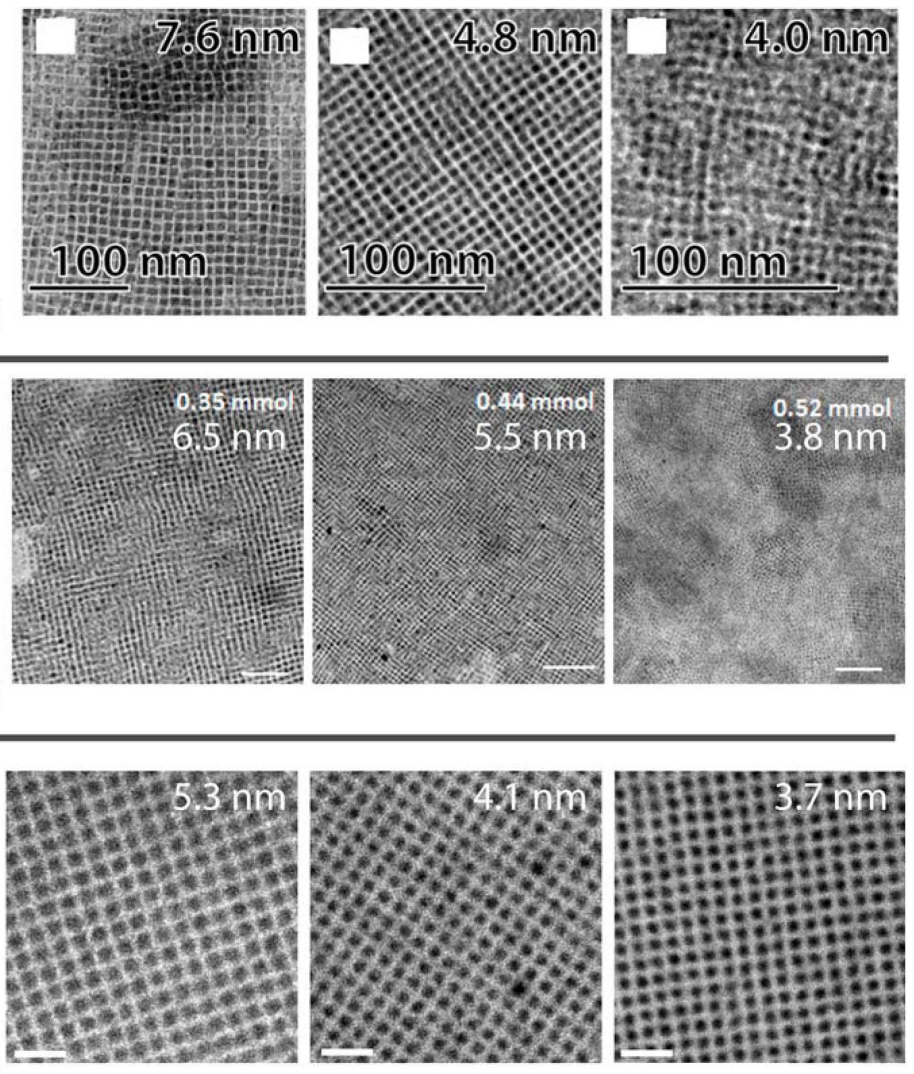

Fig. 3. (a), (b), and (c) Absorption spectra and corresponding TEM images of QDs made in Refs. 44, 45, and 46, respectively. Images were modified with permission from Refs. 44, 45, and 46 
(Fig. 3a, b) and the new method based on thermodynamic equilibrium (Fig. 3c). The exciton absorption feature in the optical spectra and the uniformity of the size in the TEM images are much greater in QDs whose size is controlled via thermodynamic equilibrium. Moreover, this method is generally applicable to the synthesis of $\mathrm{CsPbX}_{3}$ QDs of all three halides (Cl, Br, and I). Fig. 4a, b shows optical spectra and TEM images of $\mathrm{QDs}$ of $\mathrm{CsPbCl}_{3}$ and $\mathrm{CsPbI}_{3}$ prepared employing the same halide-equilibrium-based size control. Although the differences in the chemistry and kinetics of reactions involving different halide elements in the earlier synthesis methods made it difficult to generalize the synthesis protocol to all halides, it is much easier to expand the applicability of equilibrium-based synthesis.

In general, thermodynamic equilibrium is not considered a viable mechanism to control the size of nanocrystalline solids for the majority of materials. However, lead halide perovskite is a rare case in which the equilibrium approach can be used. One crucial property of $\mathrm{CsPbX}_{3} \mathrm{NCs}$ and related lead halide perovskite materials that allows the use of thermodynamic equilibrium for size control is the highly labile halide ions in the crystals. It is well known that a lead halide perovskite $\mathrm{NC}$ can undergo facile anion exchange even at room temperature ${ }^{30,31,47)}$ owing to its low kinetic barrier to diffusion in the lattice. The low kinetic barrier, especially at the elevated temperatures used in hot injection $\left(80-190^{\circ} \mathrm{C}\right)$, provides a condition in which the thermodynamic equilibrium of the halide becomes a more relevant control. The other crucial property of $\mathrm{CsPbX}_{3} \mathrm{NCs}$ is the size-dependent composition of the halide resulting from the halide-rich surface of the NCs. The relative halide content increases with decreasing QD size. This allows the equilibrium of halide ions at the interface of the NCs and the solvent medium to determine the size of the NCs. Size control was achieved either by varying the amount of halide in the reactant mixture for a given amount of $\mathrm{Cs}$ and $\mathrm{Pb}$ at a fixed reaction temperature or by varying the temperature for a fixed amount of the precursors of all three elements constituting the perovskite QDs, as shown in Fig. 4c, d, respectively. Through the law of mass action, smaller QDs were obtained when the concentration of halides increased for a fixed reaction temperature and concentrations of $\mathrm{Cs}$ and $\mathrm{Pb}$. For fixed concentrations of $\mathrm{Cs}, \mathrm{Pb}$, and $\mathrm{X}$, lowering the temperature resulted in smaller QDs. This approach produces $\mathrm{Cs}_{\mathrm{PbBr}} \mathrm{QDs}_{3}$ with high ensemble uniformity in a strongly confined regime (3.7-7 nm).

Because size control is achieved via thermodynamic equilibrium, the size of the QDs was independent of the nucleation and growth kinetics, which is one of the most distinctive and advantageous features of equilibrium-based synthesis. Fig. 5a, b shows the identical absorption and emission spectra of $\mathrm{CsPbBr}_{3}$ QDs synthesized with various reaction quenching times and precursor injection rates, which prove that size control occurs via thermodynamic equilibrium and not kinetic control. The mechanism of size control through halide equilibrium between the QD lattice and solvent medium is illustrated by the simple model shown in Fig. 5c. In this model, control of the QD size $(l)$ by varying the $\mathrm{Br}$ concentration ([Br]) can be viewed as satisfying the following equilibrium condition.

$$
\mu_{B r^{-}, s o l}\left(\left[\mathrm{Br}^{-}\right], T\right)=\mu_{B r^{-}, Q D}(l, T)
$$

$\mu_{B r^{-}, \text {sol }}\left(\left[\mathrm{Br}^{-}\right], T\right)$ is the chemical potential of $\mathrm{Br}^{-}$at a given $\left[\mathrm{Br}^{-}\right]$, value and temperature $T$ in the solution medium. $\mu_{B r^{-}, Q D}(l, T)$ is the chemical potential of $\mathrm{Br}^{-}$in the lattice of QDs with size $l$ at temperature $T$, which depends on the relative stoichiometric composition of $\mathrm{Br}^{-} \cdot \mu_{B r^{-}, \text {sol }}\left(\left[\mathrm{Br}^{-}\right], T\right)$ should increase approximately logarithmically with $\left[\mathrm{Br}^{-}\right]$ following $\mu_{B r^{-}, \text {sol }}\left(\left[\mathrm{Br}^{-}\right], T\right)=\mu_{B r^{-}, \text {sol }}^{0}+\mathrm{RT} \ln \left(\gamma\left[\mathrm{Br}^{-}\right]\right)$, where (a)

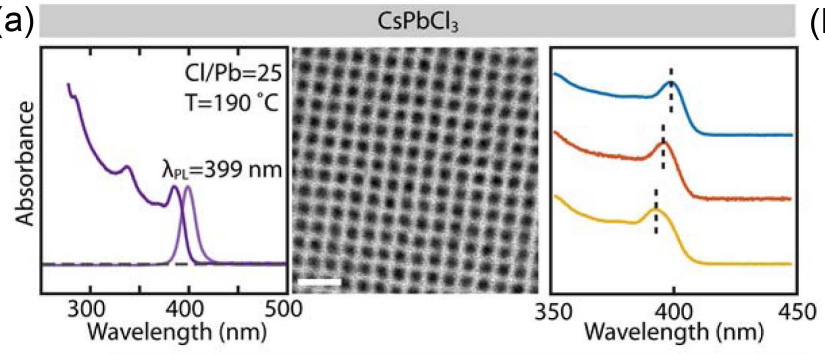

(b)

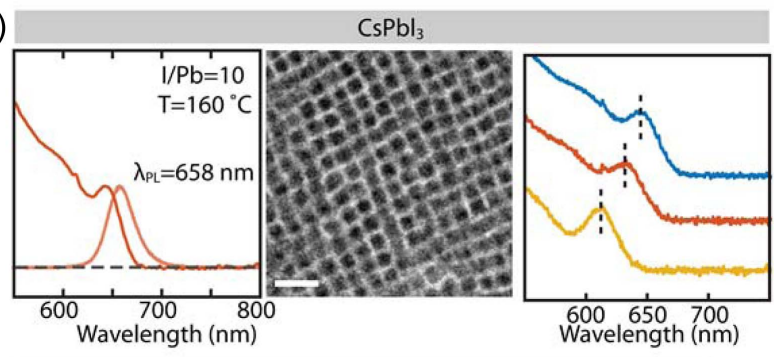

(c)

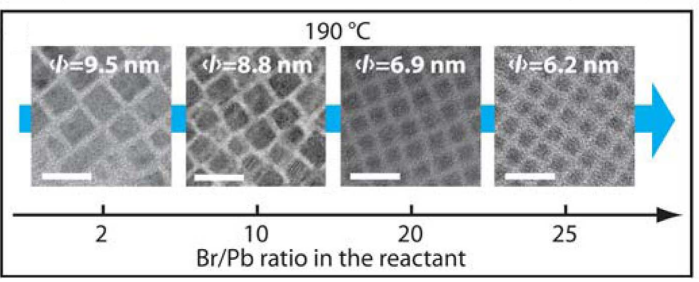

(d)

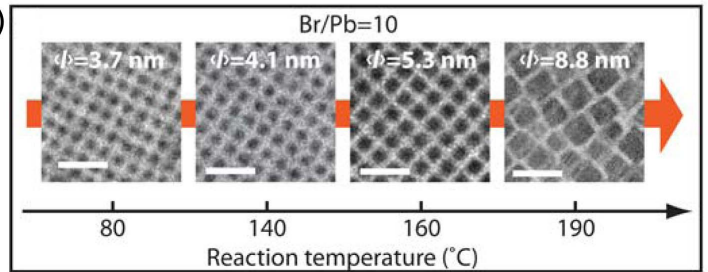

Fig. 4. Absorption and PL spectra and TEM images of (a) $\mathrm{CsPCl}_{3}$ and (b) $\mathrm{CsPbI}_{3} \mathrm{QDs}$ obtained via halide equilibrium control method, with the corresponding TEM images. The spectra show clear excitonic features, and the TEM images show size and shape uniformity. (c) Change in size of $\mathrm{CsPbBr}_{3}$ QDs with constant temperature and various $\mathrm{Br} / \mathrm{Pb}$ ratios. (d) $\mathrm{Change}$ in size of $\mathrm{CsPbrr}_{3}$ QDs with various temperatures and constant $\mathrm{Br} / \mathrm{Pb}$ ratio. Images were modified with permission from Ref. 46. 

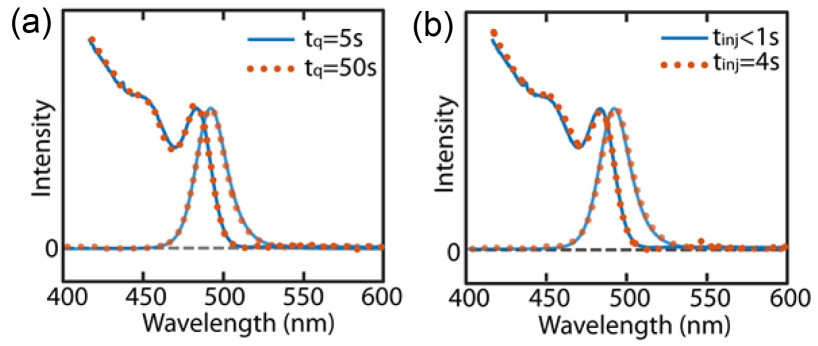

(c)

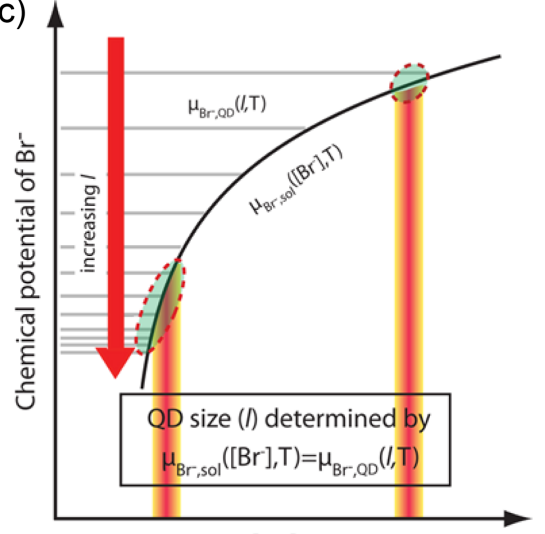

$[\mathrm{Br}]$

Fig. 5. (a) Absorption and PL spectra of $\mathrm{CsPbBr}_{3} \mathrm{NCs}$ quenched for $5 \mathrm{~s}$ (blue line) and $50 \mathrm{~s}$ (orange dashed line), (b) absorption and PL spectra of $\mathrm{CsPbBr}_{3} \mathrm{NCs}$ with cesium oleate injected swiftly (blue line) and over $4 \mathrm{~s}$ dropwise (orange dashed line). (c) Proposed mechanism of halide equilibrium control of quantum confinement in Cs-based lead halide QD synthesis. Images were modified with permission from Ref. 46.

$\mu_{B r^{-}, \text {sol }}, R$, and $\gamma$ are the chemical potential in the standard state, gas constant, and activity coefficient, respectively. The larger slope of $\mu_{B r^{-}, \text {sol }}\left(\left[\mathrm{Br}^{-}\right], T\right)$ at lower $\left(\left[\mathrm{Br}^{-}\right]\right)$and the decreasing size dependence of $\mu_{B r^{-}, Q D}(l, T)$ for larger QDs explains the less uniform size of the previously reported larger NCs synthesized by typical hot injection synthesis using a lower $\left[\mathrm{Br}^{-}\right]$value, i.e., a $\mathrm{Br} / \mathrm{Pb}$ ratio of 2 .

\section{Mn Doping of $\mathrm{CsPbX}_{3} \mathrm{NCs}$}

Isoelectronic doping of semiconductor NCs with paramag- netic transition metal ions has been extensively investigated as a way to introduce new optical, electronic, and magnetic properties through the interaction between the exciton and d electrons of the dopant ions. For instance, IIVI QDs, such as MX (where M = Cd, Zn and X=S, Se) doped with $\mathrm{Mn}^{2+}$ can generate intense sensitized dopant luminescence, creating a magnetically coupled exciton state enabling optical control of the magnetism, or produce energetic hot electrons via exciton-to-hot-carrier upconversion. $^{26,48)}$ Although it is not the focus of this article, it is worth mentioning that doping of lanthanide rare-earth metal ions has also been extensively investigated, where quantum cutting of high energy photons into low energy photons has been a topic of particular interest. ${ }^{49}$

Because $\mathrm{CsPb}_{3} \mathrm{NCs}$ were introduced as a new semiconductor NC material that can outperform many existing ones, doping of $\mathrm{Mn}^{2+}$ into these materials has been extensively attempted in recent years in anticipation of the benefits seen in other semiconductor hosts. The first successful $\mathrm{Mn}$ doping of $\mathrm{CsPbX}_{3} \mathrm{NCs}$ was reported in $\mathrm{CsPbCl}_{3}$, where hot injection synthesis of $\mathrm{CsPbCl}_{3} \mathrm{NCs}$ in the presence of an additional $\mathrm{Mn}$ precursor resulted in low-level doping of $\mathrm{Mn}$ from less than $1 \%$ up to $10 \%{ }^{27,29)}$ In this synthesis, $\mathrm{MnCl}_{2}$ was the most effective precursor of $\mathrm{Mn}$ ions, whereas many other organometallic precursors such as $\mathrm{Mn}(\mathrm{ac})_{2}, \mathrm{Mn}(\mathrm{acac})_{2}$, and $\mathrm{Mn}$ (oleate) $)_{2}$ were very ineffective as dopant precursors. When $\mathrm{MnCl}_{2}$ was used as the $\mathrm{Mn}$ precursor and $\mathrm{CsPbBr}_{3}$ was used as the host material, the resulting $\mathrm{NCs}$ were $\mathrm{Cl} / \mathrm{Br}$ mixed-halide NCs doped with $\mathrm{Mn}$. However, $\mathrm{MnBr}_{2}$ could not be used as a viable precursor of $\mathrm{Mn}$ ions for either $\mathrm{CsPbCl}_{3}$ or $\mathrm{CsPbBr}_{3}$ as the host under the hot injection synthesis condition, as will be discussed further later. Fig. $6 \mathrm{a}$ shows the absorption spectra of the undoped and $\mathrm{Mn}$ doped $\mathrm{CsPbCl}_{3}$ and $\mathrm{CsPb}(\mathrm{Cl} / \mathrm{Br})_{3}$ NCs synthesized using $\mathrm{MnCl}_{2}$ as the $\mathrm{Mn}$ precursor at a doping concentration of less than 1\%. Fig. 6b shows the corresponding luminescence spectra. In the Mn-doped NCs, characteristic Mn luminescence near $600 \mathrm{~nm}$ appeared, indicating successful doping of Mn into perovskite NC hosts with a sufficiently large bandgap, which can exhibit exciton-Mn energy transfer. The time-resolved Mn luminescence data shown in Fig. 6c can be fitted to a single exponential, indicating a relatively
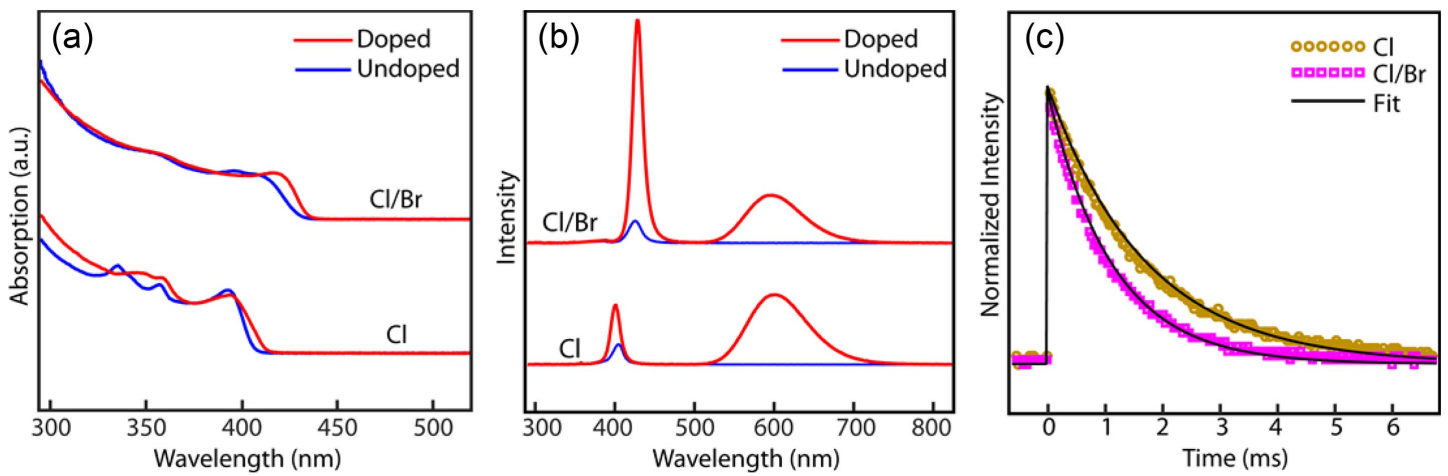

Fig. 6. (a) Absorption and (b) PL spectra of $\mathrm{Mn}$-doped $\mathrm{CsPbCl}_{3}$ and $\mathrm{CsPbCl}_{\mathrm{x}} \mathrm{Br}_{3-\mathrm{x}}$ nanocrystals with those of undoped control samples. (c) Time-dependent PL decay of Mn phosphorescence. Images were modified with permission from Ref. 27. 

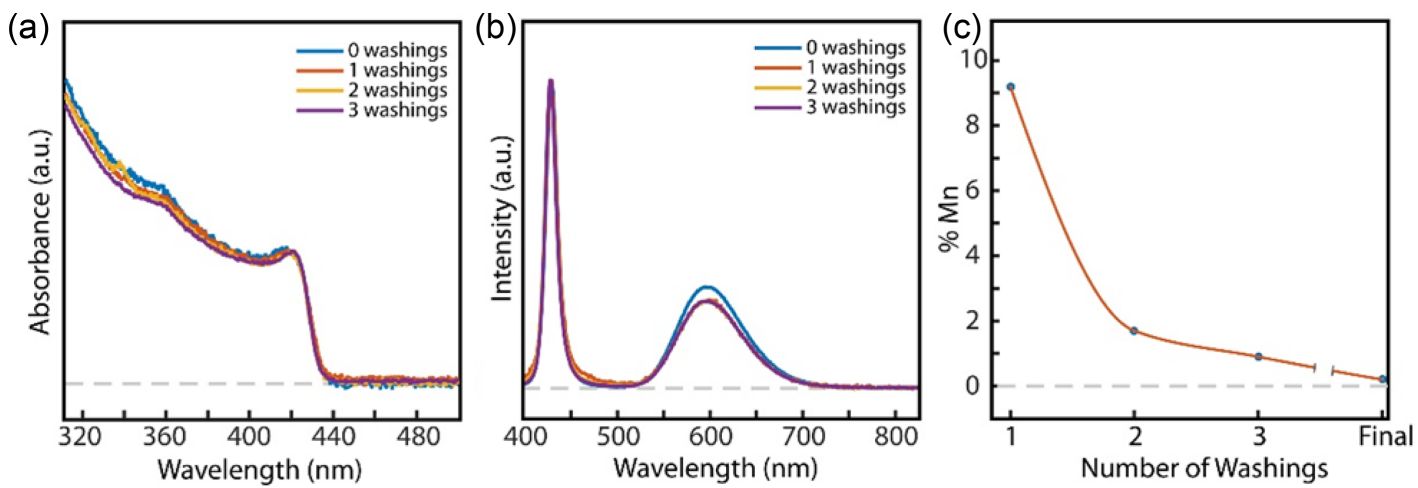

Fig. 7. (a) Absorption and (b) PL spectra of washed $\mathrm{CsPbCl}_{\mathrm{x}} \mathrm{Br}_{3-\mathrm{x}}$ sample. (c) Tested $\mathrm{Mn}$ doping concentration of samples with different numbers of washings. Images were modified with permission from Ref. 27.

homogeneous ligand field environment of the dopant ions, which is consistent with a low doping level. One of the issues in characterizing the detailed structure of Mn-doped perovskite NCs is quantifying the doping concentration, which can easily be overestimated owing to difficulties in removing the remaining $\mathrm{Mn}$ ions. Unlike many other semiconductor NCs, perovskite NCs are unstable in polar solvents, severely limiting the range of usable solvents for removing unreacted metal salts via the solvent/antisolvent approach. To overcome this issue, Son and coworkers utilized gel permeation chromatography (GPC), a technique optimized by Greytak and coworkers for cleaning II-VI $\mathrm{QDs},{ }^{50)}$ to remove the excess unreacted $\mathrm{Mn}$ and obtain a more reliable doping concentration in the thoroughly cleaned samples. Fig. 7 shows the evolution of the absorption and emission spectra as well as the measured doping concentration after each cycle of GPC purification of the NCs. After more than four GPC cycles, the measured Mn doping concentration reached a plateau, while the same absorption and emission intensity were maintained. This demonstrates clearly the necessity for thorough removal of remaining Mn ions in the solvent for accurate quantification of the doping (a)

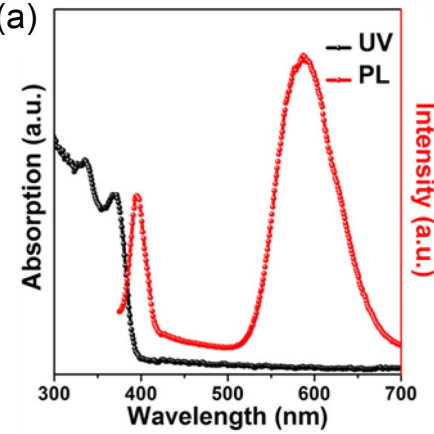

(c)

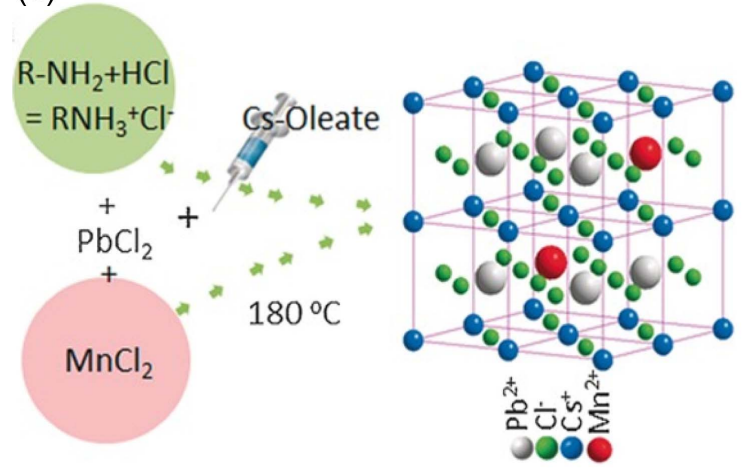

(b)

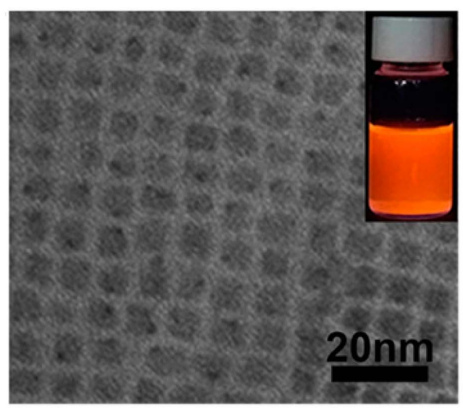

(d)

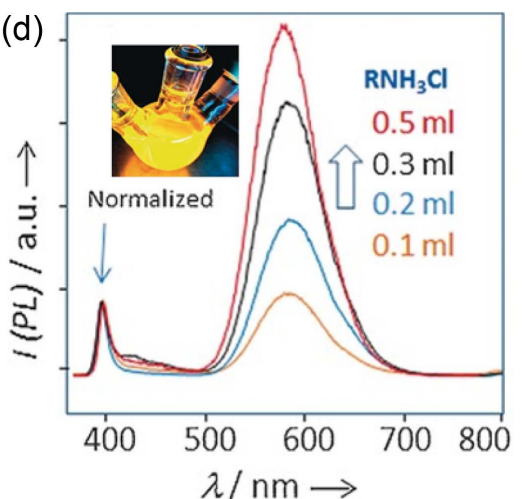

Fig. 8. (a) Absorption and PL spectra of $\mathrm{CsPbCl}_{3}$ sample with high $\mathrm{Mn}$ doping concentration ( 46\%). (b) $\mathrm{TEM}$ images of $\mathrm{CsPbCl}{ }_{3}$

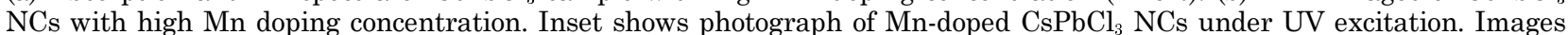
were modified with permission from Ref. 51. (c) Method to dope $\mathrm{Mn}$ in $\mathrm{CsPbCl}_{3} \mathrm{NCs}$ using an ammonium chloride mixture. (d) PL spectra of $\mathrm{CsPbCl}_{3} \mathrm{NCs}$ acquired with increasing amount of ammonium chloride mixture. Images were modified with permission from Ref. 52 . 
concentration.

In later works on $\mathrm{Mn}$-doped $\mathrm{CsPbl}_{3} \mathrm{NCs}$, the hot injection synthesis protocol was further optimized to increase the doping concentration. Yang and coworkers were able to reach a doping concentration of $46 \%$ and luminescence quantum yield of $56 \%$ by using a large amount of excess $\mathrm{Mn}$ precursors (e.g., a $\mathrm{Pb} / \mathrm{Mn}$ ratio of $1: 10){ }^{51)}$ Fig. 8a, b shows the absorption and emission spectra and TEM images of the highly $\mathrm{Mn}$-doped $\mathrm{CsPbCl}_{3} \mathrm{NCs}$. Pradhan and coworkers took another approach to increasing the efficiency of $\mathrm{Mn}$ doping, where they used oleylammonium chloride as an additional reactant that can boost the doping efficiency. They observed a large increase in the Mn luminescence intensity with increasing amount of oleylammonium chloride for a given amount of $\mathrm{Mn}$ precursor (Fig. 8c, d) ${ }^{52)}$ The authors attributed the increased efficiency of $\mathrm{Mn}$ doping to the formation of a higher concentration of NCs during the early phase of the reaction, which increased the probability of Mn doping, although the validity of this explanation is not yet fully established.

More recent synthesis of $\mathrm{Mn}$-doped $\mathrm{CsPbCl}_{3}$ has expanded the reaction conditions beyond the hot injection condition. Meijerink and coworkers showed examples of room-temperature synthesis methods for $\mathrm{Mn}$ doping of $\mathrm{CsPbCl}_{3}$ with non-halide Mn precursors. ${ }^{53)}$ In their first report, they used metal acetate salts as the precursor, which were converted to metal oleate complexes in the presence of ligands, and then added $\mathrm{HCl}$ to protonate the carboxylate group, increasing the amount of monomer initiating the formation of nanocubes. $\mathrm{HCl}$ also created a Cl-rich surface, supplying ample binding sites for $\mathrm{Mn}^{2+}$ ions and facilitating $\mathrm{Mn}$ doping. Further coating of $\mathrm{Mn}$-doped $\mathrm{CsPbCl}_{3}$ with an additional $\mathrm{CsPbCl}_{3}$ shell improved the $\mathrm{Mn}$ luminescence quantum yield.

Although earlier efforts to synthesize $\mathrm{Mn}$-doped $\mathrm{CsPbl}_{3}$ NCs produced relatively large cube-shaped NCs that experience very weak quantum confinement, several recent studies reported the synthesis of $\mathrm{CsPbCl}_{3}$ nanoplatelets with strong confinement in one dimension. Nag and coworkers synthesized $\mathrm{Mn}$-doped $\mathrm{CsPbCl}_{3}$ nanoplatelets with a thickness of $2.2 \mathrm{~nm}$, which imposes strong confinement along the thickness direction, by a room-temperature reaction. ${ }^{54)}$ They were also able to vary the doping concentration in the range of $0.2-2 \%$. Pradhan and coworkers reported another method of generating $\mathrm{Mn}$-doped $\mathrm{CsPbCl}_{3}$ nanoplatelets, which involves the initial synthesis of a Mn-doped monolayer structure and subsequent formation of nanoplatelets by the addition of Cs-oleate. ${ }^{55}$ They produced 5-nm-thick nanoplatelets with a relatively large lateral dimension in the range of 20-580 nm, which varied with the concentration of $\mathrm{Cs}$ and Mn during the reaction. Because quantum confinement of the exciton in Mn-doped semiconductor NCs can enhance the exciton-dopant interaction, which determines various magnetooptical properties, continued progress in the synthesis of strongly confined Mn-doped perovskite NCs will be important for expanding their applicability.
Most of the work on $\mathrm{Mn}$ doping of $\mathrm{CsPbX}_{3} \mathrm{NCs}$ has focused on $\mathrm{CsPbl}_{3}$ despite its less favorable optical properties, such as a higher bandgap and lower luminescence quantum yield, which are presumably due to its higher defect density compared to other halide perovskites. This is because doping of $\mathrm{Mn}$ is most favorable in $\mathrm{CsPbl}_{3}$ and becomes increasingly difficult for bromide and iodide perovskite hosts. To date, successful doping of $\mathrm{Mn}$ in a $\mathrm{CsPbBr}_{3} \mathrm{NC}$ host could not be achieved by simply extending the doping method used for producing $\mathrm{Mn}$-doped $\mathrm{CsPbCl}_{3}$ NCs. Klimov and coworkers hypothesized that direct hot injection synthesis of $\mathrm{Mn}$-doped $\mathrm{CsPbBr}_{3}$ using $\mathrm{MnBr}_{2}$ was energetically unfavorable owing to the large difference in bond energy between $\mathrm{Pb}-\mathrm{Br}(249 \mathrm{~kJ} / \mathrm{mol})$ and $\mathrm{Mn}-\mathrm{Br}(314$ $\mathrm{kJ} / \mathrm{mol})$ compared to that between $\mathrm{Pb}-\mathrm{Cl}(301 \mathrm{~kJ} / \mathrm{mol})$ and $\mathrm{Mn}-\mathrm{Cl}(338 \mathrm{~kJ} / \mathrm{mol}){ }^{29)}$ They argued that the higher stability of the $\mathrm{Mn}-\mathrm{Br}$ bond compared to the $\mathrm{Pb}-\mathrm{Br}$ bond impeded the incorporation of $\mathrm{Mn}^{2+}$ into the $\mathrm{CsPbr}_{3}$ lattice. Although it is still not clearly understood yet, the difference in the thermochemistry can certainly be a factor affecting the ability to dope $\mathrm{Mn}^{2+}$ into different $\mathrm{CsPbX}_{3} \mathrm{NCs}$.

There are several reports of attempts to utilize the ion exchange reaction to obtain $\mathrm{Mn}$-doped $\mathrm{CsPbBr}_{3} \mathrm{NCs}$. For instance, anion exchange of $\mathrm{Mn}$-doped $\mathrm{CsPbCl}_{3}$ with $\mathrm{Br}^{-}$ resulted in not only the exchange of anions but also removal of doped $\mathrm{Mn}$ ions in the host lattice. ${ }^{56)}$ Others attempted cation exchange of $\mathrm{Pb}$ with $\mathrm{Mn}$ in $\mathrm{CsPbBr}_{3}$ using $\mathrm{MnCl}_{2}$. However, this also resulted in anion exchange, ultimately forming $\mathrm{Mn}$-doped NCs with $\mathrm{Cl}^{-}$as the dominant anion in the lattice. ${ }^{57)}$ Chen and coworkers claimed that they were able to dope $\mathrm{Mn}$ into $\mathrm{CsPbBr}_{3}$ and that it increased the stability of the NCs significantly, although they did not observe Mn emission from their sample. ${ }^{57)} \mathrm{Xu}$ and Meijerink recently argued that the absence of $\mathrm{Mn}$ emission in $\mathrm{Mn}$ doped $\mathrm{CsPbBr}_{3}$ is not due to a lack of dopant ions in the lattice but due to back-energy transfer from $\mathrm{Mn}$ to the host, which effectively quenches the Mn luminescence, ${ }^{58)}$ similar to the case of Mn-doped II-VI QDs, where the bandgap of the host is not sufficiently large compared to the Mn ligand field transition energy to allow effective unidirectional energy transfer from the exciton to $\mathrm{Mn}{ }^{59)}$

Recently, Son and coworkers developed a more robust synthesis method that produces $\mathrm{Mn}$-doped $\mathrm{CsPbBr}_{3} \mathrm{NCs}$ that exhibit intense Mn luminescence. ${ }^{28)}$ The synthesis was a two-step procedure in which they first synthesized a Mndoped monolayer structure $\left(\mathrm{L}_{2}\left[\mathrm{~Pb}_{\mathrm{x}} \mathrm{Mn}_{1-\mathrm{x}} \mathrm{Br}_{4}\right]\right.$, where $\mathrm{L}$ is a ligand) as an intermediate, which they converted to Mndoped $\mathrm{CsPbBr}_{3} \mathrm{NCs}$ by adding Cs-oleate (Fig. 9a). In this method, $\mathrm{HBr}$ was used to facilitate the formation of a stable $\mathrm{L}_{2}\left[\mathrm{~Pb}_{\mathrm{x}} \mathrm{Mn}_{1-\mathrm{x}} \mathrm{Br}_{4}\right]$ monolayer structure. Mn doping of this monolayer structure was clear from the observation of intense Mn luminescence upon excitation of the host structure at $370 \mathrm{~nm}$. The absorption and emission spectra of $\mathrm{L}_{2}\left[\mathrm{~Pb}_{\mathrm{x}} \mathrm{Mn}_{1-\mathrm{x}} \mathrm{Br}_{4}\right]$ are shown in Fig. 9b, c, respectively. The absorption spectrum, which has a sharp absorption peak at $395 \mathrm{~nm}$, is very similar to that of a $\mathrm{L}_{2}\left[\mathrm{PbBr}_{4}\right]$ monolayer 
(a)

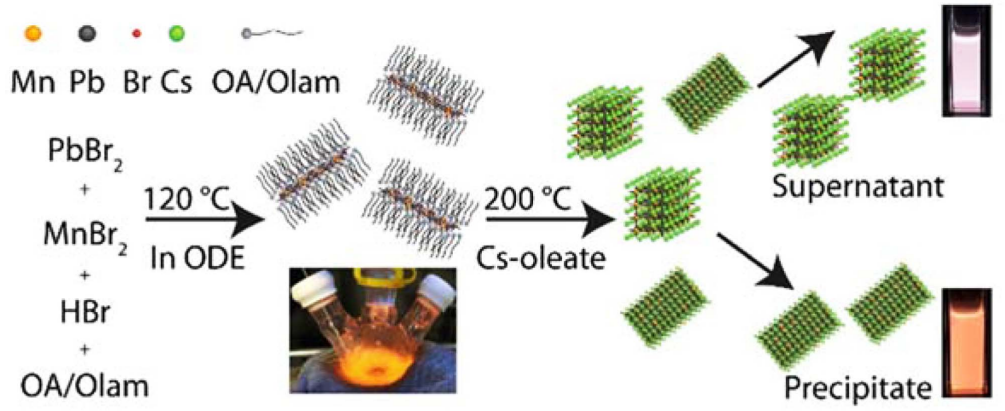

(b)

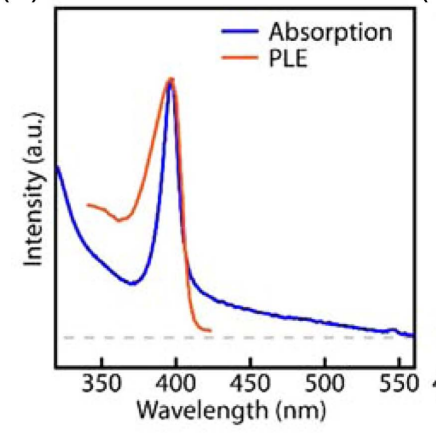

(e)

(d)

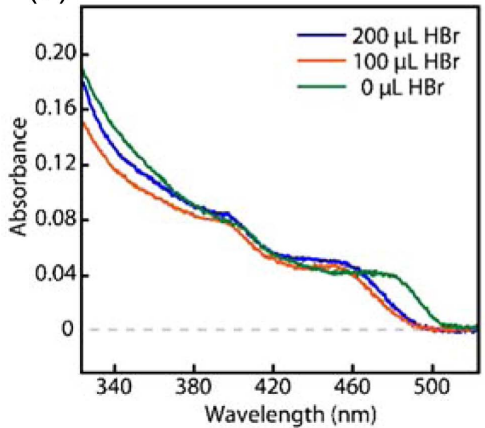

(c)



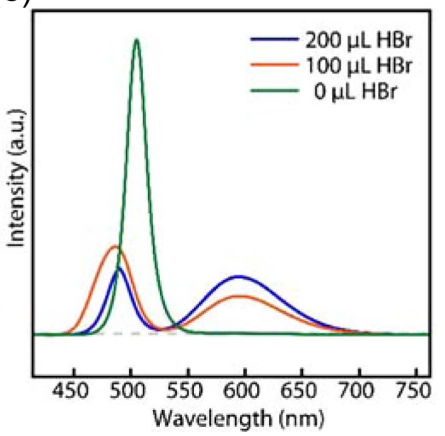

(f)

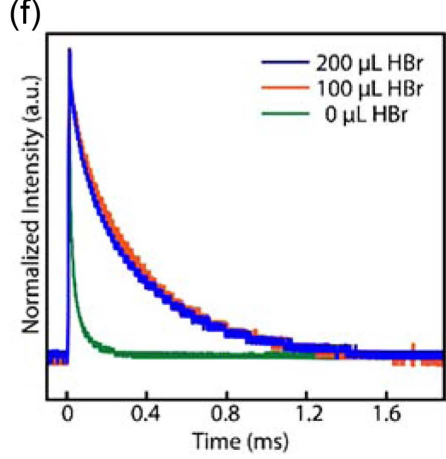

Fig. 9. (a) Schematic of Mn doping of $\mathrm{CsPbBr}_{3} \mathrm{NCs}$. (b) Absorption and (c) PL spectra of Mn-doped $\mathrm{CsPbBr}_{3} \mathrm{NCs}$ acquired with different amounts of HBr. (d) Time-dependent PL intensity of Mn phosphorescence of samples obtained with different amounts of $\mathrm{HBr}$. Images were modified with permission from Ref. 28.

reported previously, indicating that the $\mathrm{L}_{2}\left[\mathrm{~Pb}_{\mathrm{x}} \mathrm{Mn}_{1-\mathrm{x}} \mathrm{Br}_{4}\right]$ is essentially a Mn-doped monolayer structure of $\mathrm{L}_{2}\left[\mathrm{PbBr}_{4}\right]$. On the other hand, the luminescence is centered at $620 \mathrm{~nm}$ and arises entirely from Mn. The similarity of the absorption and photoluminescence excitation (PLE) spectra of $\mathrm{L}_{2}\left[\mathrm{~Pb}_{\mathrm{x}} \mathrm{Mn}_{1-\mathrm{x}} \mathrm{Br}_{4}\right]$ proved that the $\mathrm{Mn}$ luminescence in this monolayer structure is also sensitized. The intensity of the Mn luminescence in the $\mathrm{L}_{2}\left[\mathrm{~Pb}_{\mathrm{x}} \mathrm{Mn}_{1-\mathrm{x}} \mathrm{Br}_{4}\right]$ monolayer structure was well correlated with the amount of $\mathrm{HBr}$ used, which indicated that $\mathrm{HBr}$ facilitates doping of $\mathrm{Mn}$ into this structure. $\mathrm{L}_{2}\left[\mathrm{~Pb}_{\mathrm{x}} \mathrm{Mn}_{1-\mathrm{x}} \mathrm{Br}_{4}\right]$ often forms a multilayer structure when dried, as indicated by a series of small-angle diffraction peaks separated by $2 \theta=2.2^{\circ}$ in the powder X-ray diffraction patterns, which corresponds to a $4.1 \mathrm{~nm}$ spacing between the stacked layers separated by the ligand. When Cs-oleate was added to the solution of the $\mathrm{L}_{2}\left[\mathrm{~Pb}_{\mathrm{x}} \mathrm{Mn}_{1-\mathrm{x}} \mathrm{Br}_{4}\right]$ monolayer structure at $200^{\circ} \mathrm{C}, \mathrm{Mn}$-doped $\mathrm{CsPbBr}_{3} \mathrm{NCs}$ were formed. Interestingly, the resulting product was a mixture of $\mathrm{Mn}$-doped $\mathrm{CsPbBr}_{3}$ NCs with two different morphologies, i.e., nanocubes $(6.5-8.5 \mathrm{~nm})$ and nanoplatelets ( $\sim 2 \mathrm{~nm}$ in thickness). However, the two species could be readily separated via centrifugation, providing access to NCs of two different dimensions. The Mn luminescence intensity of the $\mathrm{Mn}$-doped $\mathrm{CsPbBr}_{3}$ nanocubes was well correlated with that of the $\mathrm{L}_{2}\left[\mathrm{~Pb}_{\mathrm{x}} \mathrm{Mn}_{1-\mathrm{x}} \mathrm{Br}_{4}\right]$ monolayer structure, showing an increase with the amount of $\mathrm{HBr}$ used during the initial formation of the $\mathrm{L}_{2}\left[\mathrm{~Pb}_{\mathrm{x}} \mathrm{Mn}_{1-\mathrm{x}} \mathrm{Br}_{4}\right]$ monolayer structure (Fig. 9d-f). This suggests that the formation of $\mathrm{L}_{2}\left[\mathrm{~Pb}_{1-\mathrm{x}} \mathrm{Mn}_{\mathrm{x}}\right] \mathrm{Br}_{4}$, which contains the same $\mathrm{Mn}$ $\mathrm{Br}$ coordination present in $\mathrm{Mn}$-doped $\mathrm{CsPbBr}_{3} \mathrm{NCs}$, is crucial to formation of $\mathrm{Mn}$-doped $\mathrm{CsPbBr}_{3} \mathrm{NCs}$ under a hot injection condition. The robustness of the $\mathrm{Mn}-\mathrm{Br}$ bond in $\mathrm{L}_{2}\left[\mathrm{~Pb}_{1-\mathrm{x}} \mathrm{Mn}_{\mathrm{x}}\right] \mathrm{Br}_{4}$ was demonstrated by the strong $\mathrm{Mn}$ luminescence, which survived even at $120^{\circ} \mathrm{C}$. It is also notable that unlike the $\mathrm{Mn}$-doped $\mathrm{CsPbBr}_{3}$ nanocubes, which exhibited both exciton and Mn luminescence, the Mndoped $\mathrm{CsPbBr}_{3}$ nanoplatelets exhibited primarily $\mathrm{Mn}$ luminescence, as can be determined easily from the color of 
the luminescence (Fig. 9a). This may reflect the potentially more rapid exciton-Mn energy transfer rate in the partially quantum confined nanoplatelets compared to that in the only weakly quantum confined nanocubes.

Because doping of $\mathrm{Mn}^{2+}$ ions into semiconductor $\mathrm{NCs}$ creates new optical, electronic, and magnetic properties resulting from exchange coupling between the exciton and dopant, the magnitude of the coupling is an important subject of investigation. It was explored quite extensively in II-VI Mn-doped semiconductor NCs as a function of the structural parameters, which affect the spatial overlap of the exciton and dopant wavefunction dictating the strength of the exchange coupling of the exciton and dopant. ${ }^{26,60,61)}$ For the Mn-doped $\mathrm{CsPbX}_{3} \mathrm{NC}$ system, Son and coworkers performed the first experiment to provide useful information on the strength of the exciton-dopant exchange coupling in comparison to that in Mn-doped II-VI semiconductor NCs. ${ }^{62)}$ The approach taken was to compare the exciton-Mn energy transfer times $\left(\tau_{\mathrm{ET}}\right)$ of $\mathrm{Mn}$-doped $\mathrm{CsPbCl}_{3} \mathrm{NCs}$ and $\mathrm{Mn}$ doped CdS/ZnS NCs after normalizing to the doping concentration. In their study, $\tau_{\mathrm{ET}}$ was obtained by comparative analysis of the exciton relaxation dynamics in Mn-doped and undoped NCs using pump-probe transient absorption spectroscopy to monitor the dynamics of exciton relaxation. The energy transfer pathway that exists only in Mn-doped NCs was manifested as an additional dynamic component in the bleach recovery of the exciton, as shown in Fig. 10a. In $\mathrm{Mn}$-doped $\mathrm{CsPbCl}_{3}$ nanocubes with an edge length of 10 $\mathrm{nm}$ and $\mathrm{a} \sim 0.4 \%$ doping concentration, $\tau_{\mathrm{ET}}$ was determined to be $\sim 380 \mathrm{ps}^{62)}$ Compared to the $\tau_{\mathrm{ET}}$ value of the previously studied Mn-doped CdS/ZnS NCs after correcting for the difference in doping concentration, the $\tau_{\mathrm{ET}}$ in the Mn-doped $\mathrm{CsPbCl}_{3}$ nanocubes is $2-5$ times slower. The authors concluded that although this may suggest that the excitonMn exchange interaction is weaker than that in the II-VI semiconductor NC host, the significant difference in the quantum confinement in the two NC samples should also be considered to make a more meaningful comparison. Because the exciton in $\mathrm{Mn}$-doped $\mathrm{CsPbCl}_{3}$ nanocubes with an edge length of $10 \mathrm{~nm}$ is very weakly confined, in contrast to that in the much smaller Mn-doped CdS/ZnS NCs, the comparison of $\tau_{\mathrm{ET}}$ in their study may underestimate the relative

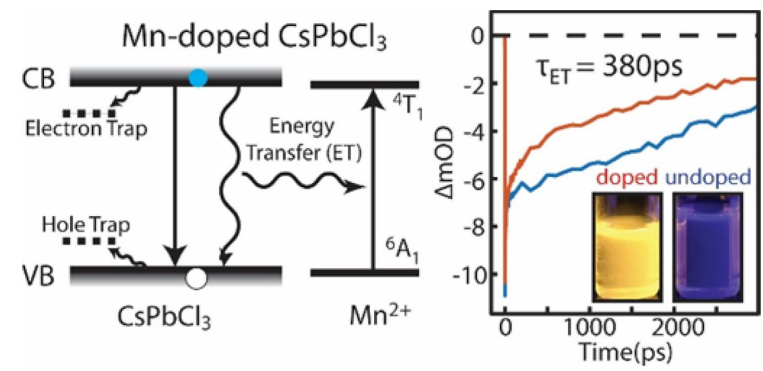

Fig. 10. Scheme of exciton-to-dopant energy transfer in Mndoped $\mathrm{CsPbCl}_{3} \mathrm{NCs}$ and transient absorption data for doped and undoped $\mathrm{CsPCl}_{3} \mathrm{NCs}$. Images were modified with permission from Ref. 62 . strength of exciton-Mn coupling in Mn-doped $\mathrm{CsPbCl}_{3}$ nanocubes. Clearly, further investigation of other Mn-doped $\mathrm{CsPbX}_{3} \mathrm{NCs}$ with different halide compositions and varying degrees of quantum confinement is necessary to obtain a better picture of the coupling between the exciton and dopant in this system.

\section{Outlook and Conclusion}

Although the first chemical synthesis of colloidal $\mathrm{CsPbX}_{3}$ perovskite NCs was reported only several years ago, much progress has been made since then in both the synthesis and characterization of $\mathrm{CsPbX}_{3} \mathrm{NCs}$ with much higher control of the dimensions and chemical composition. One of the most important achievements in the synthesis of $\mathrm{CsPbX}_{3}$ perovskite NCs was the development of methods to precisely control the quantum confinement with very high ensemble uniformity of the morphology and size in the strongly confined regime. This opened the door to exploitation of quantum confinement of excitons in perovskite NCs for their application, which had been lagging compared to that of other semiconductor NCs. Another notable achievement in the synthesis of colloidal $\mathrm{CsPbX}_{3}$ perovskite NCs was successful doping with paramagnetic transition metal ions such as $\mathrm{Mn}^{2+}$. This provided access to various new optical, electronic, and magnetic properties originating from the coupling of the exciton and magnetic dopant ions that are not present in undoped perovskite NCs. Although the ability to control the quantum confinement and introduce magnetic dopants into the lattice of $\mathrm{CsPbX}_{3} \mathrm{NCs}$ was acquired only recently, methodologies have rapidly expanded to $\mathrm{CsP}$ $\mathrm{bX}_{3} \mathrm{NCs}$ of different dimensions beyond simple nanocubes. With continuing development of the synthesis of $\mathrm{CsPbX}_{3}$ NCs with higher levels of structural and chemical control, it will also be necessary to explore the structure-property relationship and use the new properties in applications.

\section{Acknowledgments}

This work was supported by the Welch Foundation (A1639). D.P. was supported by the NSF GRFP (DGE1252521).

\section{REFERENCES}

1. X. Zhang, H. Lin, H. Huang, C. Reckmeier, Y. Zhang, W. C. H. Choy, and A. L. Rogach, "Enhancing the Brightness of Cesium Lead Halide Perovskite Nanocrystal Based Green Light-Emitting Devices through the Interface Engineering with Perfluorinated Ionomer," Nano Lett., 16 [2] 1415-20 (2016).

2. G. Li, F. W. R. Rivarola, N. J. L. K. Davis, S. Bai, T. C. Jellicoe, F. de la Peña, S. Hou, C. Ducati, F. Gao, R. H. Friend, N. C. Greenham, and Z.-K. Tan, "Highly Efficient Perovskite Nanocrystal Light-Emitting Diodes Enabled by a Universal Crosslinking Method," Adv. Mater., 28 [18] 3528-34 (2016) 
3. Q. A. Akkerman, M. Gandini, F. Di Stasio, P. Rastogi, F. Palazon, G. Bertoni, J. M. Ball, M. Prato, A. Petrozza, and L. Manna, "Strongly Emissive Perovskite Nanocrystal Inks for High-Voltage Solar Cells," Nat. Energy 216194 (2016).

4. A. Swarnkar, A. R. Marshall, E. M. Sanehira, B. D. Chernomordik, and D. T. Moore, J. A. Christians, T. Chakrabarti, and J. M. Luther, "Quantum Dot-Induced Phase Stabilization of a-CsPbI ${ }_{3}$ Perovskite for High-Efficiency Photovoltaics," Science, 354 [6308] 92-5 (2016).

5. A. Kojima, K. Teshima, Y. Shirai, and T. Miyasaka, "Organometal Halide Perovskites as Visible-Light Sensitizers for Photovoltaic Cells," J. Am. Chem. Soc. 131 [17] 6050-51 (2009).

6. G. R. Yettapu, D. Talukdar, S. Sarkar, A. Swarnkar, A. Nag, P. Ghosh, and P. Mandal, "Terahertz Conductivity within Colloidal $\mathrm{CsPbBr}_{3}$ Perovskite Nanocrystals: Remarkably High Carrier Mobilities and Large Diffusion Lengths," Nano Lett., 16 [8] 4838-48 (2016).

7. S. ten Brinck and I. Infante, "Surface Termination, Morphology, and Bright Photoluminescence of Cesium Lead Halide Perovskite Nanocrystals," ACS Energy Lett., 1 [6] 1266-72 (2016).

8. R. E. Brandt, J. R. Poindexter, P. Gorai, R. C. Kurchin, R. L. Z. Hoye, L. Nienhaus, M. W. B. Wilson, J. A. Polizzotti, R. Sereika, R. Žaltauskas, L. C. Lee, J. L. MacManusDriscoll, M. Bawendi, V. Stevanović, and T. Buonassisi, "Searching for "Defect-Tolerant" Photovoltaic Materials: Combined Theoretical and Experimental Screening," Chem. Mater., 29 [11] 4667-74 (2017).

9. L. Protesescu, S. Yakunin, M. I. Bodnarchuk, F. Krieg, R. Caputo, C. H. Hendon, R. X. Yang, A. Walsh, and M. V. Kovalenko, "Nanocrystals of Cesium Lead Halide Perovskites $\left(\mathrm{CsPbX}_{3}, \mathrm{X}=\mathrm{Cl}, \mathrm{Br}\right.$, and I): Novel Optoelectronic Materials Showing Bright Emission with Wide Color Gamut," Nano Lett., 15 [6] 3692-96 (2015).

10. P. K. Santra and P. V. Kamat, "Mn-Doped Quantum Dot Sensitized Solar Cells: A Strategy to Boost Efficiency over 5\%," J. Am. Chem. Soc., 134 [5] 2508-11 (2012)

11. P. V. Kamat, "Quantum Dot Solar Cells. The Next Big Thing in Photovoltaics," J. Phys. Chem. Lett., 4 [6] 908-18 (2013).

12. A. J. Nozik, "Quantum Dot Solar Cells," Phys. E, 14 [1-2] 115-20 (2002).

13. A. H. Ip, S. M. Thon, S. Hoogland, O. Voznyy, D. Zhitomirsky, R. Debnath, L. Levina, L. R. Rollny, G. H. Carey, A. Fischer, K. W. Kemp, I. J. Kramer, Z. Ning, A. J. Labelle, K. W. Chou, A. Amassian, and E. H. Sargent, "Hybrid Passivated Colloidal Quantum Dot Solids," Nat. Nanotechnol., 7577 (2012).

14. I. Gur, N. A. Fromer, M. L. Geier, and A. P. Alivisatos, "Air-Stable All-Inorganic Nanocrystal Solar Cells Processed from Solution," Science, 310 [5747] 462-5 (2005).

15. J.-H. Choi, H. Wang, S. J. Oh, T. Paik, P. Sung, J. Sung, X. Ye, T. Zhao, B. T. Diroll, C. B. Murray, C. R. Kagan, "Exploiting the Colloidal Nanocrystal Library to Construct Electronic Devices," Science, 352 [6282] 205-8 (2016).

16. C. R. Kagan, E. Lifshitz, E. H. Sargent, and D. V. Talapin, "Building Devices from Colloidal Quantum Dots," Science,
353 [6302] aac5523 (2016).

17. S. A. McDonald, G. Konstantatos, S. Zhang, P. W. Cyr, E. J. D. Klem, L. Levina, E. H. Sargent, "Solution-Processed PbS Quantum Dot Infrared Photodetectors and Photovoltaics," Nat. Mater., 4138 (2005).

18. X. Michalet, F. F. Pinaud, L. A. Bentolila, J. M. Tsay, S. Doose, J. J. Li, G. Sundaresan, A. M. Wu, S. S. Gambhir, and S. Weiss, "Quantum Dots for Live Cells, in vivo Imaging, and Diagnostics," Science 307 [5709] 538-44 (2005).

19. I. L. Medintz, H. T. Uyeda, E. R. Goldman, and H. Mattoussi, "Quantum Dot Bioconjugates for Imaging, Labelling and Sensing," Nat. Mater., 4 [6] 435-46 (2005).

20. X. H. Gao, Y. Y. Cui, R. M. Levenson, L. W. K. Chung, and S. M. Nie, "In vivo Cancer Targeting and Imaging with Semiconductor Quantum Dots," Nat. Biotechnol., 22 [8] 969-76 (2004).

21. A. P. Alivisatos, "Semiconductor Clusters, Nanocrystals, and Quantum Dots," Science, 271 [5251] 933-37 (1996).

22. D. J. Norris, N. Yao, F. T. Charnock, and T. A. Kennedy, "High-Quality Manganese-Doped ZnSe Nanocrystals," Nano Lett., 1 [1] 3-7 (2001).

23. D. J. Norris, A. L. Efros, and S. C. Erwin, "Doped Nanocrystals," Science, 319 [5871] 1776-79 (2008).

24. N. Pradhan and X. G. Peng, "Efficient and Color-Tunable Mn-Doped ZnSe Nanocrystal Emitters: Control of Optical Performance via Greener Synthetic Chemistry," J. Am. Chem. Soc., 129 [11] 3339-47 (2007).

25. B. B. Srivastava, S. Jana, N. S. Karan, S. Paria, N. R. Jana, D. D. Sarma, and N. Pradhan, "Highly Luminescent Mn-Doped ZnS Nanocrystals: Gram-Scale Synthesis," J. Phys. Chem. Lett., 1 [9] 1454-58 (2010).

26. R. Beaulac, L. Schneider, P. I. Archer, G. Bacher, and D. R. Gamelin, "Light-Induced Spontaneous Magnetization in Doped Colloidal Quantum Dots," Science, 325 [5943] 973-76 (2009).

27. D. Parobek, B. J. Roman, Y. Dong, H. Jin, E. Lee, M. Sheldon, and D. H. Son, "Exciton-to-Dopant Energy Transfer in Mn-Doped Cesium Lead Halide Perovskite Nanocrystals," Nano Lett., 16 [12] 7376-80 (2016).

28. D. Parobek, Y. Dong, T. Qiao, and D. H. Son, "Direct HotInjection Synthesis of Mn-Doped CsPbBr $\mathrm{Can}_{3}$ Nanocrystals," Chem. Mater., 30 [9] 2939-44 (2018).

29. W. Liu, Q. Lin, H. Li, K. Wu, I. Robel, J. M. Pietryga, and V. I. Klimov, " $\mathrm{Mn}^{2+}$-Doped Lead Halide Perovskite Nanocrystals with Dual-Color Emission Controlled by Halide Content," J. Am. Chem. Soc., 138 [45] 14954-61 (2016).

30. Q. A. Akkerman, V. D'Innocenzo, S. Accornero, A. Scarpellini, A. Petrozza, M. Prato, and L. Manna, "Tuning the Optical Properties of Cesium Lead Halide Perovskite Nanocrystals by Anion Exchange Reactions," J. Am. Chem. Soc., 137 [32] 10276-81 (2015).

31. G. Nedelcu, L. Protesescu, S. Yakunin, M. I. Bodnarchuk, M. J. Grotevent, and M. V. Kovalenko, "Fast AnionExchange in Highly Luminescent Nanocrystals of Cesium Lead Halide Perovskites $\left(\mathrm{CsPbX}_{3}, \mathrm{X}=\mathrm{Cl}, \mathrm{Br}, \mathrm{I}\right)$," Nano Lett., 15 [8] 5635-40 (2015).

32. J. Li, L. Luo, H. Huang, C. Ma, Z. Ye, J. Zeng, and H. He, "2D Behaviors of Excitons in Cesium Lead Halide Perovskite Nanoplatelets," J. Phys. Chem. Lett., 8 [6] 1161- 
68 (2017).

33. J. Aneesh, A. Swarnkar, V. Kumar Ravi, R. Sharma, A. Nag, and K. V. Adarsh, "Ultrafast Exciton Dynamics in Colloidal $\mathrm{CsPbBr}_{3}$ Perovskite Nanocrystals: Biexciton Effect and Auger Recombination," J. Phys. Chem. C, 121 [8] 4734-39 (2017).

34. A. Shinde, R. Gahlaut, and S. Mahamuni, "Low-Temperature Photoluminescence Studies of $\mathrm{CsPbBr}_{3}$ Quantum Dots," J. Phys. Chem. C, 121 [27] 14872-78 (2017).

35. H. Utzat, K. E. Shulenberger, O. B. Achorn, M. Nasilowski, T. S. Sinclair, and M. G. Bawendi, "Probing Linewidths and Biexciton Quantum Yields of Single Cesium Lead Halide Nanocrystals in Solution," Nano Lett., 17 [11] 6838-46 (2017).

36. M. A. Becker, R. Vaxenburg, G. Nedelcu, P. C. Sercel, A. Shabaev, M. J. Mehl, J. G. Michopoulos, S. G. Lambrakos, N. Bernstein, J. L. Lyons, T. Stöferle, R. F. Mahrt, M. V. Kovalenko, D. J. Norris, G. Rainò, and A. L. Efros, "Bright Triplet Excitons in Caesium Lead Halide Perovskites," Nature, 553 189-93 (2018).

37. M. C. Brennan, J. E. Herr, T. S. Nguyen-Beck, J. Zinna, S. Draguta, S. Rouvimov, J. Parkhill, and M. Kuno, "Origin of the Size-Dependent Stokes Shift in $\mathrm{CsPbBr}_{3}$ Perovskite Nanocrystals," J. Am. Chem. Soc., 139 [35] 12201-8 (2017).

38. J. Chen, K. Žídek, P. Chábera, D. Liu, P. Cheng, L. Nuuttila, M. J. Al-Marri, H. Lehtivuori, M. E. Messing, K. Han, K. Zheng, and T. Pullerits, "Size- and Wavelength-Dependent Two-Photon Absorption Cross-Section of $\mathrm{CsPbBr}_{3}$ Perovskite Quantum Dots," J. Phys. Chem. Lett., 8 [10] 2316-21 (2017).

39. M. Koolyk, D. Amgar, S. Aharon, and L. Etgar, "Kinetics of Cesium Lead Halide Perovskite Nanoparticle Growth; Focusing and De-Focusing of Size Distribution," Nanoscale, 8 [12] 6403-9 (2016).

40. Y. Bekenstein, B. A. Koscher, S. W. Eaton, P. Yang, and A. P. Alivisatos, "Highly Luminescent Colloidal Nanoplates of Perovskite Cesium Lead Halide and Their Oriented Assemblies," J. Am. Chem. Soc., 137 [51] 16008-11 (2015).

41. Q. A. Akkerman, S. G. Motti, A. R. Srimath Kandada, E. Mosconi, V. D’Innocenzo, G. Bertoni, S. Marras, B. A. Kamino, L. Miranda, F. De Angelis, A. Petrozza, M. Prato, and L. Manna, "Solution Synthesis Approach to Colloidal Cesium Lead Halide Perovskite Nanoplatelets with Monolayer-Level Thickness Control," J. Am. Chem. Soc., 138 [3] 1010-16 (2016)

42. A. Pan, B. He, X. Fan, Z. Liu, J. J. Urban, A. P. Alivisatos, L. He, and Y. Liu, "Insight into the Ligand-Mediated Synthesis of Colloidal $\mathrm{CsPbBr}_{3}$ Perovskite Nanocrystals: The Role of Organic Acid, Base, and Cesium Precursors," ACS Nano, 10 [8] 7943-54 (2016).

43. S. Sun, D. Yuan, Y. Xu, A. Wang, and Z. Deng, "LigandMediated Synthesis of Shape-Controlled Cesium Lead Halide Perovskite Nanocrystals via Reprecipitation Process at Room Temperature," ACS Nano, 10 [3] 3648-57 (2016).

44. G. Almeida, L. Goldoni, Q. Akkerman, Z. Dang, A. H. Khan, S. Marras, I. Moreels, L. Manna, "Role of AcidBase Equilibria in the Size, Shape, and Phase Control of
Cesium Lead Bromide Nanocrystals," ACS Nano, 12 [2] 1704-11 (2018).

45. A. Dutta, S. K. Dutta, S. Das Adhikari, and N. Pradhan, "Tuning the Size of $\mathrm{CsPbBr}_{3}$ Nanocrystals: All at One Constant Temperature," ACS Energy Lett., 3 [2] 329-34 (2018).

46. Y. Dong, T. Qiao, D. Kim, D. Parobek, D. Rossi, and D. H. Son, "Precise Control of Quantum Confinement in Cesium Lead Halide Perovskite Quantum Dots via Thermodynamic Equilibrium," Nano Lett., 18 [6] 3716-22 (2018).

47. D. Parobek, Y. Dong, T. Qiao, D. Rossi, and D. H. Son, "Photoinduced Anion Exchange in Cesium Lead Halide Perovskite Nanocrystals," J. Am. Chem. Soc., 139 [12] 4358-61 (2017)

48. Y. Dong, J. Choi, H.-K. Jeong, and D. H. Son, "Hot Electrons Generated from Doped Quantum Dots via Upconversion of Excitons to Hot Charge Carriers for Enhanced Photocatalysis," J. Am. Chem. Soc., 137 [16] 5549-54 (2015)

49. G. Pan, X. Bai, D. Yang, X. Chen, P. Jing, S. Qu, L. Zhang, D. Zhou, J. Zhu, W. Xu, B. Dong, and H. Song, "Doping Lanthanide into Perovskite Nanocrystals: Highly Improved and Expanded Optical Properties," Nano Lett., 17 [12] 8005-11 (2017).

50. Y. Shen, M. Y. Gee, R. Tan, P. J. Pellechia, and A. B. Greytak, "Purification of Quantum Dots by Gel Permeation Chromatography and the Effect of Excess Ligands on Shell Growth and Ligand Exchange," Chem. Mater., 25 [14] 2838-48 (2013).

51. H. Liu, Z. Wu, J. Shao, D. Yao, H. Gao, Y. Liu, W. Yu, H. Zhang, and B. Yang, " $\mathrm{CsPb} \mathrm{B}_{\mathrm{x}} \mathrm{Mn}_{1-\mathrm{x}} \mathrm{Cl}_{3}$ Perovskite Quantum Dots with High Mn Substitution Ratio," ACS Nano, 11 [2] 2239-47 (2017).

52. S. Das Adhikari, S. K. Dutta, A. Dutta, A. K. Guria, and N. Pradhan, "Chemically Tailoring the Dopant Emission in Manganese-Doped $\mathrm{CsPbCl}_{3}$ Perovskite Nanocrystals," Angew. Chem. Int. Ed., 56 [30], 8746-50 (2017).

53. K. Xu, C. C. Lin, X. Xie, and A. Meijerink, "Efficient and Stable Luminescence from $\mathrm{Mn}^{2+}$ in Core and Core-Isocrystalline Shell $\mathrm{CsPbCl}_{3}$ Perovskite Nanocrystals," Chem. Mater., 29 [10] 4265-72 (2017).

54. W. J. Mir, M. Jagadeeswararao, S. Das, and A. Nag, "Colloidal Mn-Doped Cesium Lead Halide Perovskite Nanoplatelets," ACS Energy Lett., 2 [3] 537-43 (2017).

55. S. Das Adhikari, A. Dutta, S. K. Dutta, and N. Pradhan, "Layered Perovskites $\mathrm{L}_{2}\left(\mathrm{~Pb}_{1-\mathrm{x}} \mathrm{Mn}_{\mathrm{x}}\right) \mathrm{Cl}_{4}$ to $\mathrm{Mn}$-Doped $\mathrm{CsPb}$ $\mathrm{Cl}_{3}$ Perovskite Platelets," ACS Energy Lett., 3 [6] 1247-53 (2018).

56. F. Li, Z. Xia, C. Pan, Y. Gong, L. Gu, Q. Liu, and J. Z. Zhang, "High $\mathrm{Br}^{-}$Content $\mathrm{CsPb}\left(\mathrm{Cl}_{\mathrm{y}} \mathrm{Br}_{1-\mathrm{y}}\right)_{3}$ Perovskite Nanocrystals with Strong $\mathrm{Mn}^{2+}$ Emission through Diverse Cation/Anion Exchange Engineering," ACS Appl. Mater. Interfaces, 10 [14] 11739-46 (2018).

57. S. Zou, Y. Liu, J. Li, C. Liu, R. Feng, F. Jiang, Y. Li, J. Song, H. Zeng, M. Hong, and X. Chen, "Stabilizing Cesium Lead Halide Perovskite Lattice through Mn(II) Substitution for Air-Stable Light-Emitting Diodes," J. Am. Chem. Soc., 139 [33] 11443-50 (2017).

58. K. Xu and A. Meijerink, "Tuning Exciton- $\mathrm{Mn}^{2+}$ Energy 
Transfer in Mixed Halide Perovskite Nanocrystals," Chem. Mater., 30 [15] 5346-52 (2018).

59. R. Beaulac, P. I. Archer, J. van Rijssel, A. Meijerink, and D. R. Gamelin, "Exciton Storage by $\mathrm{Mn}^{2+}$ in Colloidal $\mathrm{Mn}^{2+}$ Doped CdSe Quantum Dots," Nano Lett., 8 [9] 2949-53 (2008).

60. H.-Y. Chen, S. Maiti, and D. H. Son, "Doping LocationDependent Energy Transfer Dynamics in Mn-Doped CdS/ ZnS Nanocrystals," ACS Nano, 6 [1] 583-91 (2012).
61. W. D. Rice, W. Liu, V. Pinchetti, D. R. Yakovlev, V. I. Klimov, and S. A. Crooker, "Direct Measurements of Magnetic Polarons in $\mathrm{Cd}_{1-\mathrm{x}} \mathrm{Mn}_{\mathrm{x}}$ Se Nanocrystals from Resonant Photoluminescence," Nano Lett., 17 [5] 3068-75 (2017).

62. D. Rossi, D. Parobek, Y. Dong, and D. H. Son, "Dynamics of Exciton-Mn Energy Transfer in Mn-Doped $\mathrm{CsPbCl}_{3}$ Perovskite Nanocrystals,” J. Phys. Chem. C, 121 [32] 17143-49 (2017). 